\title{
Veterans' Continued Participation in an Annual Fecal Immunochemical Test Mailing Program for Colorectal Cancer Screening
}

\author{
Jennifer A. Schlichting, PhD, Michelle A. Mengeling, PhD, Nader M. Makki, MD, \\ Ashish Malhotra, MD, Thorvardur R. Halfdanarson, MD, J. Stacey Klutts, MD, PhD, \\ Barcey T. Levy, MD, PhD, Peter J. Kaboli, MD, MS, and Mary E. Charlton, RN, PhD
}

Objective: The objective of this study was to determine what proportion of veterans previously screened for colorectal cancer (CRC) using fecal immunochemical testing (FIT) would be willing to undergo a second round of FIT screening.

Methods: Patients in the Iowa City Veterans Affairs Health Care System ( $<65$ years old, asymptomatic, average risk, overdue for CRC screening) who completed a mailed FIT (April 2011 to May 2012) were contacted 1 year later by telephone to collect demographic and recent CRC screening information, and were offered a second mailed FIT if eligible.

Results: Of 204 veterans who completed initial FIT testing, 159 were eligible to participate in a second round of FIT screening; $132(83 \%)$ participated in the telephone survey, and $126(79 \%)$ completed a second annual FIT, with $10(8 \%)$ individuals testing positive. The majority of participants $(67 \%)$ reported being more likely to take a yearly FIT than a colonoscopy every 10 years. Participants overwhelmingly reported that the FIT was easy to use and convenient (89\%), and they were likely to complete a mailed FIT each year (97\%).

Conclusions: Those willing to take a mailed FIT seem satisfied with this method and willing to do it annually. Population-based or provider-based FIT mailing programs have the potential to increase CRC screening in overdue populations. (J Am Board Fam Med 2015;28:494-497.)

Keywords: Cancer Screening, Colorectal Cancer

The most recent US Preventive Services Task Force colorectal cancer (CRC) screening guidelines recommend average-risk adults aged 50 to 75 years receive colonoscopy every 10 years; sigmoidoscopy every 5 years, with a midinterval high-sensitivity

\footnotetext{
This article was externally peer reviewed.

Submitted 25 August 2014; revised 29 December 2014; accepted 9 January 2015.

From the VA Office of Rural Health, Rural Health Resource Center, Central Region, Iowa City, IA (JAS, MAM, AM, PJK, MEC); the Comprehensive Access and Delivery Research and Evaluation (CADRE) Center and Pathology and Laboratory Medicine (JSK), Iowa City VA Healthcare System, Iowa City, IA (JAS, MAM, AM, PJK, MEC); the Department of Epidemiology, University of Iowa College of Public Health, Iowa City (JAS, BTL, MEC); the Division of General Internal Medicine, Department of Internal Medicine (MAM, NMM, PJK), the Department of Pathology (JSK), and the Department of Family Medicine (BTL), University of Iowa Carver College of Medicine, Iowa City; and the Division of Oncology, Department of Internal Medicine, Mayo Clinic, Scottsdale, AZ (TRH).
}

fecal occult blood test (FOBT) every 3 years; or annual high-sensitivity FOBT or fecal immunochemical testing (FIT). ${ }^{1}$ Several barriers to colonoscopy, such as distance from health care facilities and transportation to and from the facility, have

Funding: This research was supported by the Department of Veterans Affairs, Veterans Health Administration, Office of Rural Health, Veterans Rural Health Resource CenterCentral Region (VRHRC-CR), and the Veterans Affairs Health Services Research and Development (HSR\&D) Service, Center for Comprehensive Access and Delivery Research and Evaluation (CADRE) (CIN 13-412). This work was also supported by the University of Iowa Holden Comprehensive Cancer Center, which is funded in part by NIH/ NCI P30 CA086862.

Conflict of interest: none declared.

Disclaimer: The views expressed in this article are those of the authors and do not necessarily represent the views or policy of the Department of Veterans Affairs or the United States Government.

Corresponding author: Mary E. Charlton, RN, PhD, Iowa City VA Health Care System, 601 Hwy 6 West, Iowa City, IA 52246 (E-mail: mary.charlton@va.gov). 
been reported. ${ }^{2,3}$ These issues are particularly relevant to the US Department of Veterans Affairs Health Administration (VHA) because over 3 million $(36 \%)$ of enrolled veterans reside in rural areas. $^{4}$

The VHA recently approved the use of FIT, a high-sensitivity FOBT that can be administered at home. Advantages of FIT over guaiac FOBT include higher sensitivity for detection of significant adenomas and CRC, lack of diet or medication restrictions, and the need for only 1 sample., ${ }^{2,3}$

We previously demonstrated the effectiveness of an initial mailing of a FIT to eligible patients overdue for CRC screening. ${ }^{2,3}$ Although annual FIT screening is recommended, little is known regarding patients' willingness to participate in repeated annual FIT screenings. Therefore, the objective of this study was to determine what proportion of patients who previously screened negative with FIT would be willing to complete a second round of annual FIT screening, and what proportion would screen positive.
The 2 mailed FIT interventions have been described previously. ${ }^{2,3}$ In the initial 2 interventions, 232 participants completed a FIT. Of these participants, $28(12 \%)$ had positive FITs and were referred for follow-up care, whereas those who tested negative $(n=204)$ were included in this prospective follow-up study to determine their willingness to complete a second annual FIT.

Approximately 1 year after completing their initial FIT, potentially eligible patients received a recruitment letter explaining the study, followed by a telephone call to assess eligibility and willingness to take a brief survey. Immediately following the survey, participants were asked to take a second annual FIT. Those who agreed were mailed an OC FIT-CHEK kit (Polymedco, Cortlandt Manor, $\mathrm{NY}$ ) with instructions and a preaddressed, postagepaid envelope to return the FIT.

Of those eligible $(\mathrm{n}=159), 132(83 \%)$ participated in the survey and $126(79 \%)$ returned a second annual FIT. There were 10 positive FITs $(8 \%)$, and these patients were referred for fol-

Figure 1. Study population, Iowa City VA Health Care System. *Percentage calculated from the total eligible to participate. Of 204 invited to participate, 45 were ineligible ( 2 moved out of the area, 1 was deceased, 17 selfreported scheduled colonoscopy or otherwise being up to date on screening, and 25 reported new symptoms and/ or family history on the eligibility questionnaire given before the survey). This left 159 potentially eligible to participate. Three were unable to be contacted and 24 declined participation, but were included in these 159 potentially eligible patients. FIT, fecal immunochemical testing.

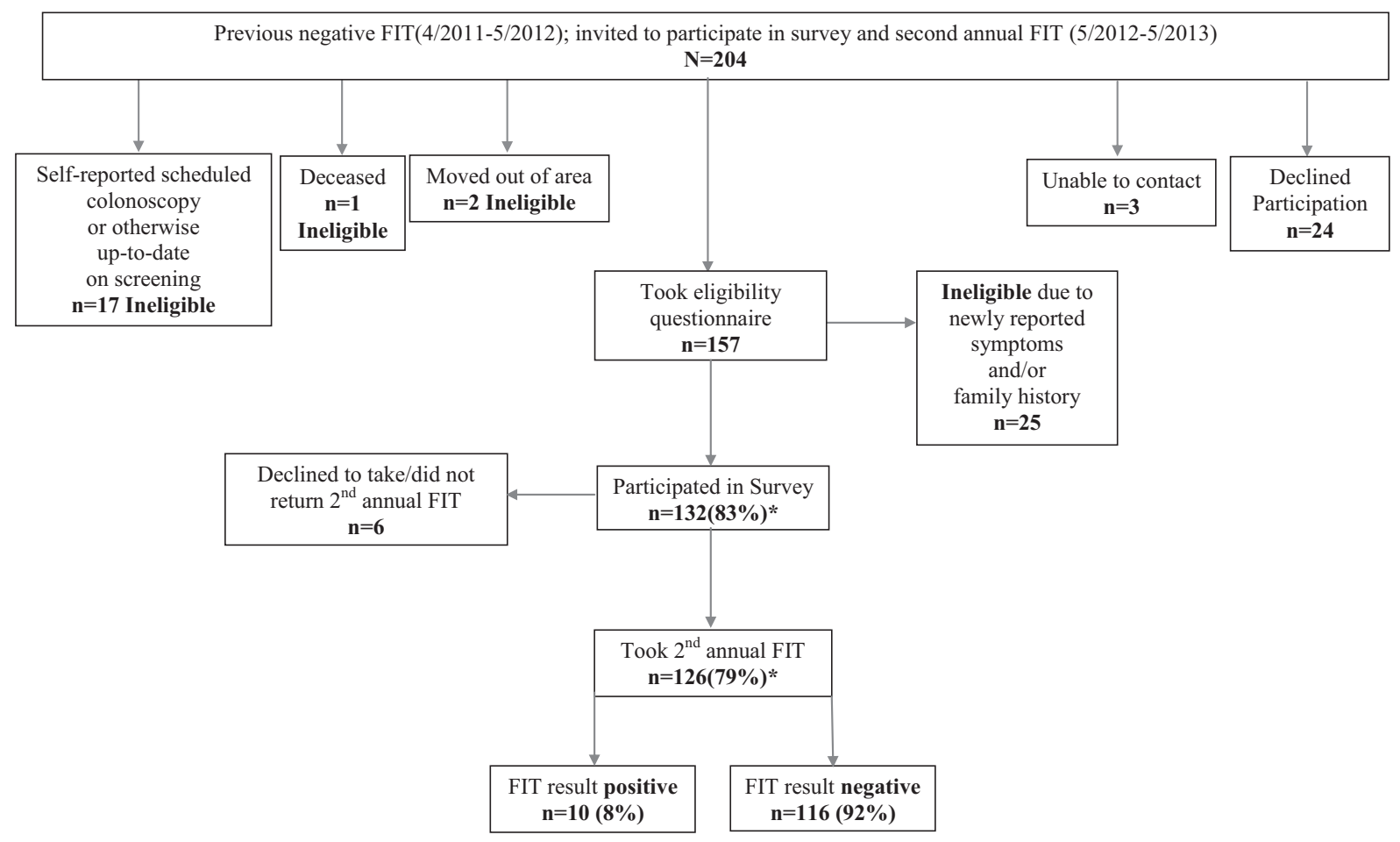


Table 1. Survey Participant Characteristics and Responses, Iowa City VA Health Care System $(n=132)$

\begin{tabular}{|c|c|}
\hline Characteristics/Preferences, 2012 to 2013 & Participants \\
\hline Age in years (mean, SD) & $62(4)$ \\
\hline Male sex & $122(92)$ \\
\hline Rural area of residence & $78(59)$ \\
\hline Good/very good/excellent general health & $95(72)$ \\
\hline \multicolumn{2}{|l|}{ Race } \\
\hline White & $123(93)$ \\
\hline Black & $6(5)$ \\
\hline Other & $3(2)$ \\
\hline \multicolumn{2}{|l|}{ Health insurance in addition to VHA benefits (could report $>1$ ) } \\
\hline Private individual or group plan & $34(26)$ \\
\hline Military & $17(13)$ \\
\hline Medicaid & $8(6)$ \\
\hline Medicare & $31(23)$ \\
\hline VHA benefits only & $65(49)$ \\
\hline Highest education more than some college or technical school & $74(56)$ \\
\hline Have at least 1 child ( $\leq 18$ years) living in household & $17(13)$ \\
\hline More than 1 adult ( $>18$ years) living in household & $89(67)$ \\
\hline Currently married/partnered & $77(58)$ \\
\hline Participant has someone who can bring to health care appointments if needed & $121(92)$ \\
\hline Has an additional non-VA primary care provider & $30(23)$ \\
\hline Health care provider has previously recommended a colonoscopy & $95(72)$ \\
\hline Ever had a colonoscopy & $60(45)$ \\
\hline Ever had colonoscopy performed at a VA facility & $12(20)$ \\
\hline \multicolumn{2}{|l|}{ Who should make the decision for when you should be screened for colon cancer?* } \\
\hline You alone or mostly you & $21(16)$ \\
\hline The doctor and you equally & $77(58)$ \\
\hline Mostly the doctor or the doctor alone & $33(25)$ \\
\hline \multicolumn{2}{|l|}{ Who should make the decision for which test should be used for screening?* } \\
\hline You alone or mostly you & $37(28)$ \\
\hline The doctor and you equally & $35(26)$ \\
\hline Mostly the doctor or the doctor alone & $59(45)$ \\
\hline \multicolumn{2}{|l|}{ More likely to take colonoscopy every 10 years or FIT every year? } \\
\hline FIT & $88(67)$ \\
\hline Colonoscopy & $11(8)$ \\
\hline No preference & $32(24)$ \\
\hline Prefer not to be screened & $1(1)$ \\
\hline \multicolumn{2}{|l|}{ Preferred method to receive CRC screening due reminders (could report $>1$ ) } \\
\hline Mail & $110(83)$ \\
\hline Phone & $55(42)$ \\
\hline E-mail & $30(23)$ \\
\hline At clinic visit & $40(30)$ \\
\hline No reminder & $1(1)$ \\
\hline \multicolumn{2}{|l|}{ Preferred method to receive FIT kit } \\
\hline Mail & $120(91)$ \\
\hline Pick up at nearest VA facility & $1(1)$ \\
\hline No preference & $11(8)$ \\
\hline \multicolumn{2}{|l|}{ FIT satisfaction (answered "extremely" or "very") } \\
\hline How easy was it for you to follow the FIT kit directions? & $117(89)$ \\
\hline How convenient was the FIT kit for you? & $118(89)$ \\
\hline If the FIT was mailed to you each year, how likely would you be to complete it each year? & $128(97)$ \\
\hline
\end{tabular}

Data are $\mathrm{n}(\%)$ unless otherwise indicated.

${ }^{*}$ One missing response.

CRC, colorectal cancer; FIT, fecal immunochemical testing; SD, standard deviation; VHA, Veterans Health Administration. 
low-up care (Figure 1). Participants overwhelmingly reported the FIT was easy to use and convenient $(89 \%)$, and they would be likely to complete a mailed FIT each year (97\%) (Table 1). There were no significant differences between FIT participants versus nonparticipants by age, sex, or rurality (data not shown).

Few studies have examined patients' willingness to participate in follow-up FIT after their initial screening. In this group of largely rural veterans initially overdue for CRC screening, 79\% participated in a second annual mailed FIT. Similarly, a population-based FIT screening program in Amsterdam demonstrated that $85 \%$ of participants completed a second biennial mailed FIT. ${ }^{5}$

Several limitations should be considered when interpreting the study results. Because of the limited sample size, we were unable to conduct subgroup analyses. Although the original cohort was identified based on regular VHA use, 17 participants reported scheduled or up-to-date CRC screens from outside the VHA, which we were unable to verify. Furthermore, this sample of veterans who previously participated in a FIT intervention may not be generalizable to other clinic populations. This is, however, one of the few studies to present data on repeated FIT testing in the United States.

\section{Conclusion}

FIT mailing programs seem to be an effective method to provide CRC screening for overdue patients, especially those who face barriers to colonoscopy. Providers should consider offering FIT as an option along with other evidence-based methods.

The authors thank Stacy Wittrock and Ashley Cozad for their efforts in coordinating this study. The authors also acknowledge Lindsey Davenport-Landry in the Iowa City Veterans Affairs laboratory for her work managing all the laboratory aspects of this project.

\section{References}

1. United States Preventive Services Task Force. Screening for colorectal cancer: U.S. Preventive Services Task Force recommendation statement. Ann Intern Med 2008;149:627-37.

2. Charlton ME, Mengeling MA, Halfdanarson TR, et al. Evaluation of a home-based colorectal cancer screening intervention in a rural state. J Rural Health 2014;303:322-32.

3. Schlichting JA, Mengeling MA, Makki NM, et al. Increasing colorectal cancer screening in an overdue population: participation and cost impacts of adding telephone calls to a FIT mailing program. J Community Health 2014;39:239-47.

4. Fact sheet: information about the VHA Office of Rural Health and Rural Veterans. Washington, DC: Veterans Health Administration, Office of Rural Health; 2014. Available from: http://www. ruralhealth.va.gov/docs/factsheets/ORH_General_ FactSheet_2014.pdf. Accessed December 29, 2014.

5. Denters MJ, Deutekom M, Bossuyt PM, van Rijn AF, Fockens P, Dekker E. Involvement of previous nonparticipants cannot fully compensate for lower participation in a second round of FIT-screening. Cancer Epidemiol 2013;37:330-5. 